Supporting Information for:

\title{
Two-Dimensional SiS Layers with Promising Electronic and Optoelectronic Properties: Theoretical Prediction
}

Ji-Hui Yang, ${ }^{*} \dagger, \|$ Yueyu Zhang, ${ }^{\ddagger}, \S$ Wan-Jian Yin, ${ }^{\dagger}$ X. G. Gong, ${ }^{\star}{ }^{\S}$ Boris I. Yakobson,, and Su-Huai $\mathrm{Wei}^{*}{ }{ }_{\perp}$

${ }^{\dagger}$ National Renewable Energy Laboratory, Golden, Colorado 80401, United States

${ }^{*}$ Key Laboratory for Computational Physical Sciences (MOE), State Key Laboratory of Surface

Physics, Department of Physics,Fudan University, Shanghai 200433, China

${ }^{\S}$ Collaborative Innovation Center of Advanced Microstructures, Nanjing 210093, China

"Department of Materials Science and NanoEngineering, Rice University, Houston, Texas 77005, United States

${ }^{\perp}$ Beijing Computational Science Research Center, Beijing 100094, China 


\section{Atomic Coordinates}

\section{Pmma-SiS}

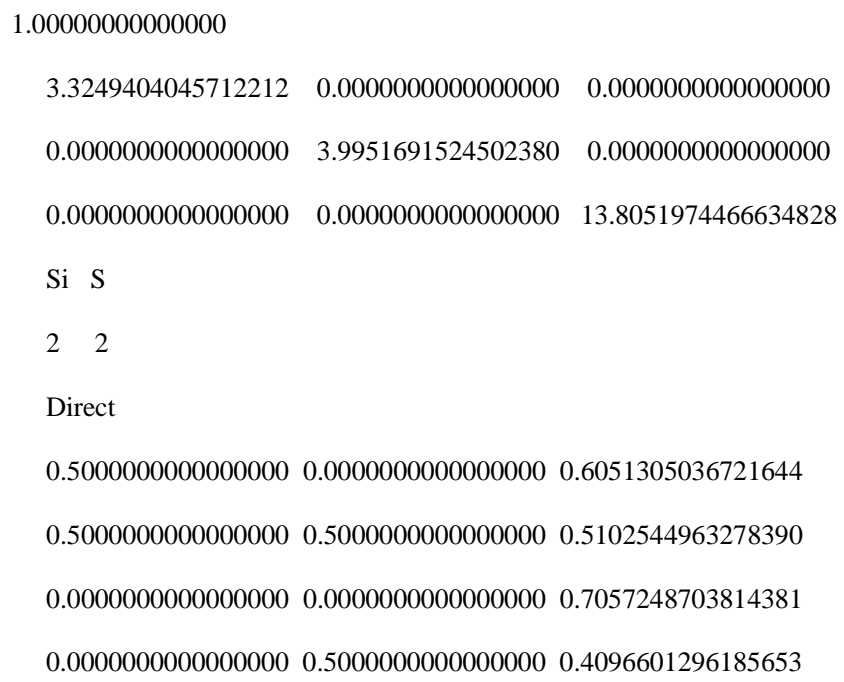

\section{Pma2-SiS}

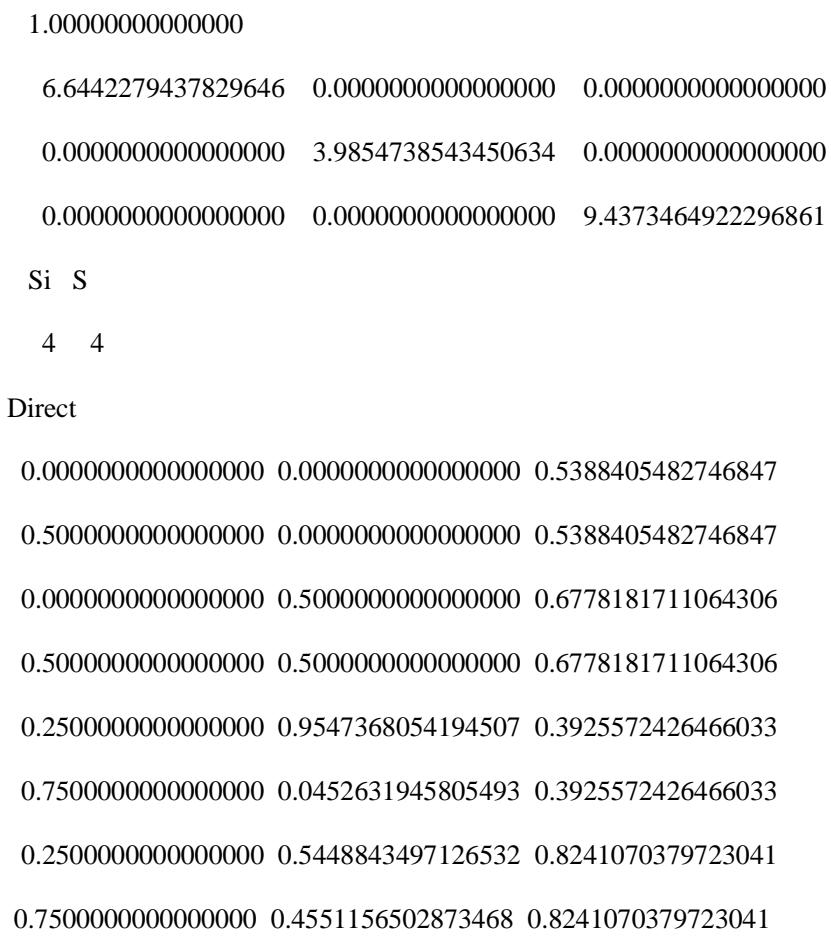




\title{
silicene sulfide
}

\author{
1.00000000000000 \\ $4.5631999968999999 \quad 0.0000000000000000 \quad 0.0000000000000000$ \\ $1.7632501853000000 \quad 3.6032041415000000 \quad 0.0000000000000000$ \\ $\begin{array}{llll}0.0000000000000000 & 0.0000000000000000 & 13.3178997040000002\end{array}$ \\ Si S \\ 22 \\ Direct \\ $0.34049999700000200 .8297500010000007 \quad 0.5000000000000000$ \\ $\begin{array}{lll}0.6595000029999980 & 0.1702499989999993 & 0.5000000000000000\end{array}$ \\ $0.0000000000000000 \quad 0.0000000000000000 .6249099970000032$ \\ $0.0000000000000000 \quad 0.00000000000000000 .3750900029999968$
}




\section{Phonon Spectra}
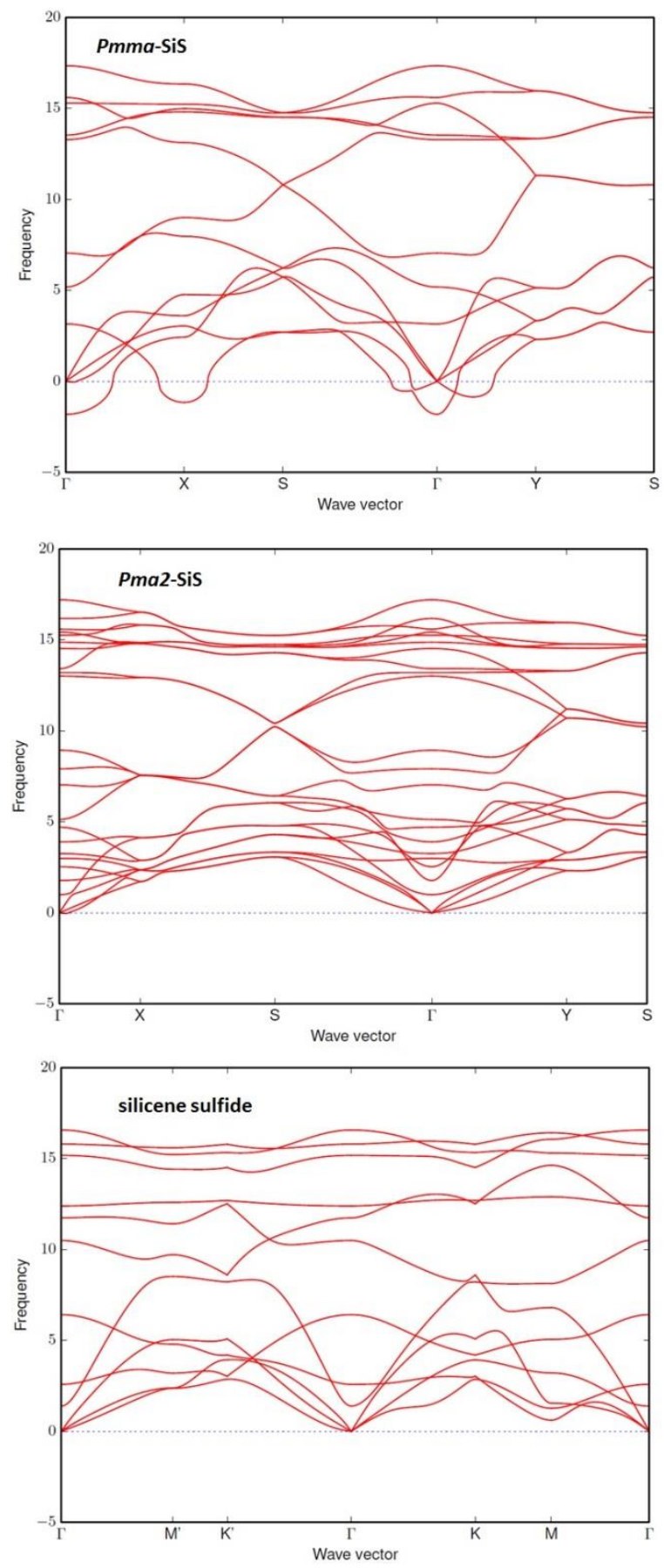

Fig. S1. Phonon spectra of Pmma-SiS, Pma2-SiS, and silicene sulfide. The imaginary frequency at X point in Pmma-SiS indicates it's not dynamically stable. Both Pma2-SiS and silicene sulfide show no imaginary frequency, suggesting they are dynamically stable. 


\section{Molecular Dynamical Simulations for Pma2-SiS and silicene sulfide}

To study the thermal stability of Pma2-SiS and silicene sulfide, we performed molecular dynamical simulations for these two structures at $\mathrm{T}=300 \mathrm{~K}$ and $500 \mathrm{~K}$. As shown in Fig. S2, Pma2-SiS remains stable at $\mathrm{T}=300 \mathrm{~K}$ with a converged total energy. At $\mathrm{T}=500 \mathrm{~K}$, the structure still shows good shape (see Fig. S2(e)), but the total energy is not converged yet in our simulation time (8ps). For silicene sulfide, the structure shows some buckling with some broken bonds (see Fig. S2(i)), which is expected from the buckling structure of pure silicene. Although the total energy is not converged, the structure shape also doesn't change much at both $\mathrm{T}=300 \mathrm{~K}$ and $\mathrm{T}=500 \mathrm{~K}$. 
(a)

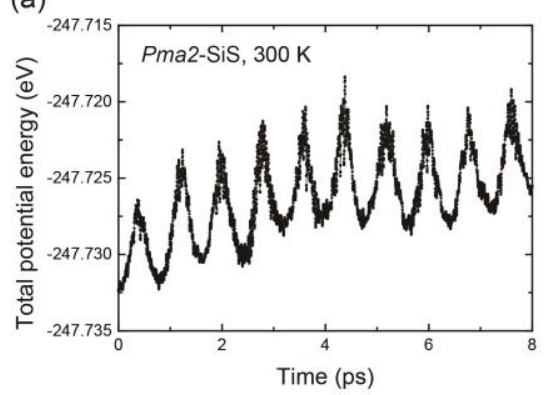

(c)

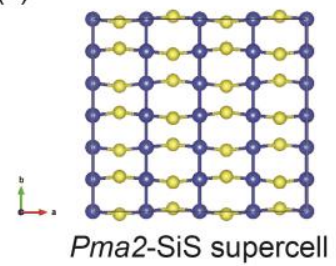

(f)

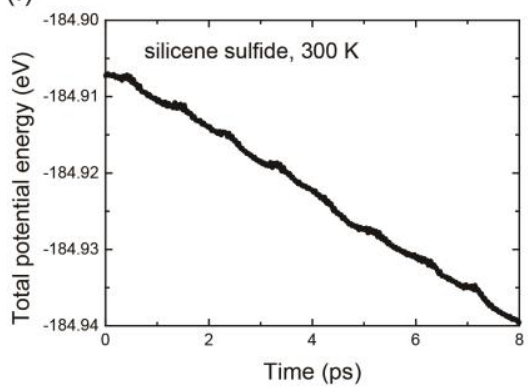

(h)

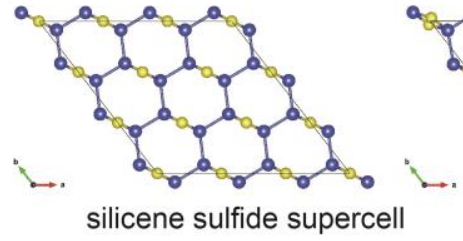

(b)

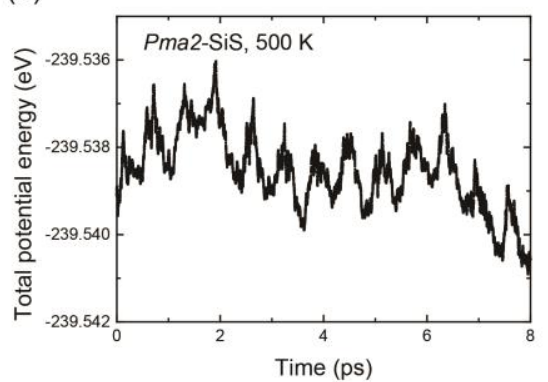

(e)

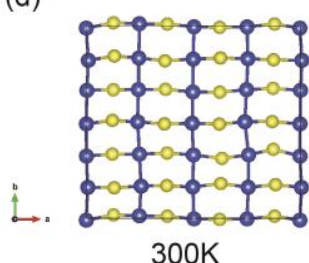

(g)

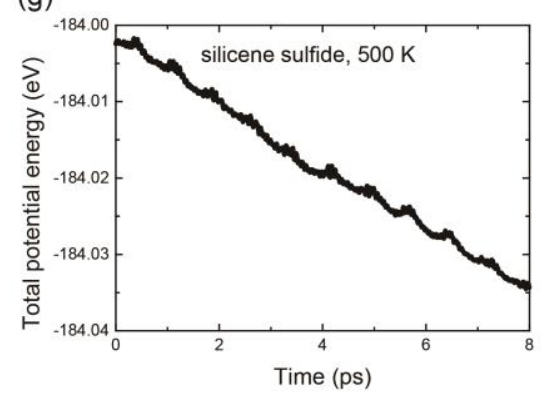

(j)

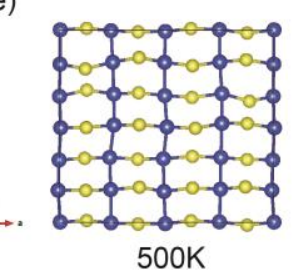

(i)

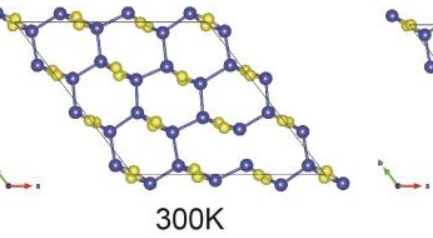

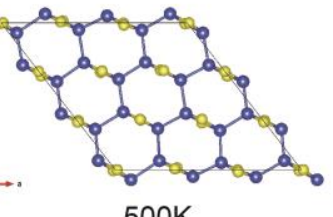

Fig. S2. (a) The fluctuation of total energy of Pma2-SiS supercell (48 atoms/cell) as a function of the molecular dynamic simulation step at $300 \mathrm{~K}$ and (b) $500 \mathrm{~K}$. (c) The supercell of Pma2-SiS used in the molecular dynamic simulation, and a snapshot of the system at $300 \mathrm{~K}$ (d) and $500 \mathrm{~K}$ (e). (f) The fluctuation of total energy of silicene sulfide supercell (36 atoms/cell) as a function of the molecular dynamic simulation step at $300 \mathrm{~K}$ and $(\mathrm{g}) 500 \mathrm{~K}$. (h) The supercell of silicene sulfide used in the molecular dynamic simulation, and a snapshot of the system at $300 \mathrm{~K}$ (i) and $500 \mathrm{~K}(\mathrm{j})$. 


\section{Quasiparticle $G_{0} W_{0}$ calculations of bandgaps for Pma2-SiS and silicene sulfide}

To confirm the optical bandgaps of Pma2-SiS and silicene sulfide, we did $G_{0} W_{0}$ calculations using VASP. We use 200 bands and 100 bands for Pma2-SiS and silicene sulfide and NOMEGA is set 100 . To obtain the quasiparticle bandgaps, we did three $G_{0} W_{0}$ calculations for each structure with different vacuum lengths and then extrapolate the quasiparticle bandgaps when the vacuum lengths go to infinity, as was done in Phys. Rev. Lett. 2015, 115, 066403. As shown in Fig. S3, the quasiparticle bandgaps of Pma2-SiS and silicene sulfide are $2.22 \mathrm{eV}$ and $1.95 \mathrm{eV}$, respectively. The exciton binding energies are linearly related to the quasiparticle bandgaps, which are $E_{b}=\alpha E_{g}+b$, as shown in Phys. Rev. Lett. 2015, 115, 066403, where $\alpha=0.21$ and $\beta=0.40$. As a result, the exciton binding energies of Pma2-SiS and silicene sulfide are $0.87 \mathrm{eV}$ and $0.81 \mathrm{eV}$, respectively. Consequently, the optical bandgaps of of Pma2SiS and silicene sulfide are $1.35 \mathrm{eV}$ and $1.14 \mathrm{eV}$, respectively.
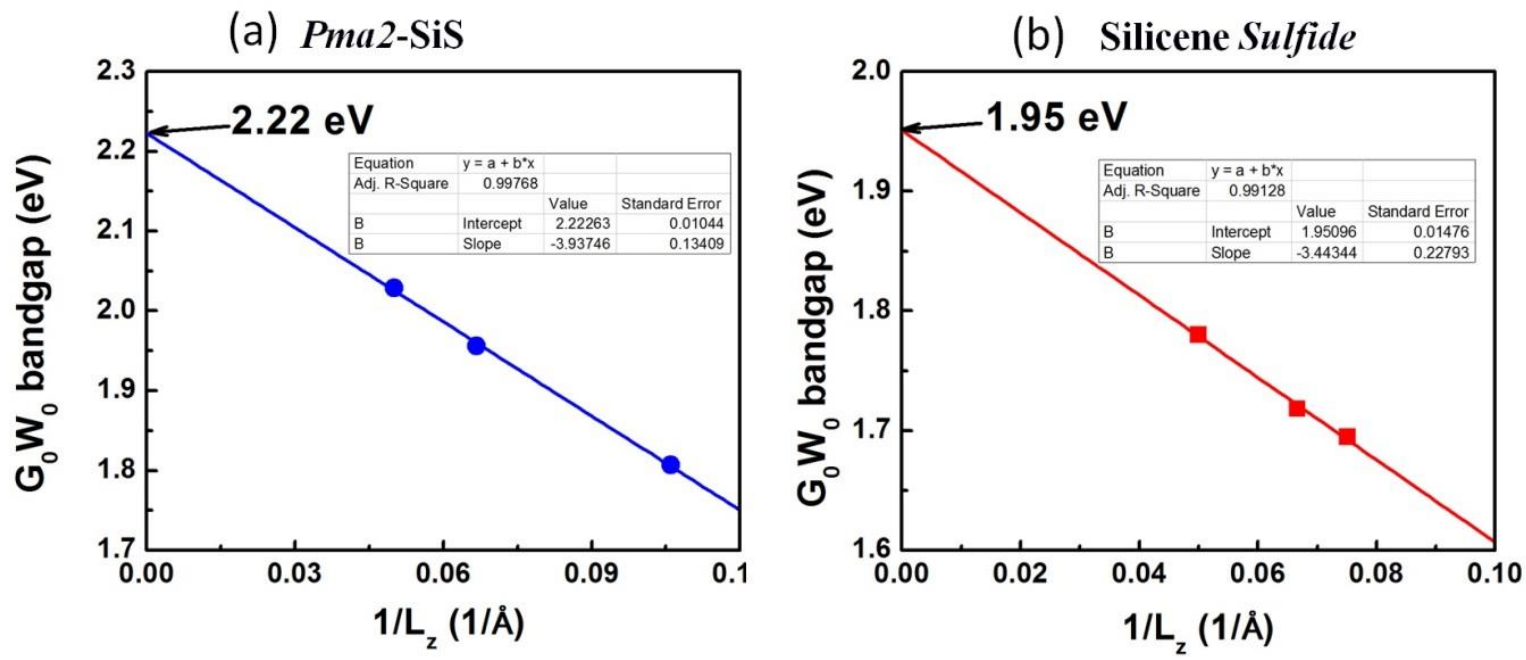

Fig. S3. $\mathrm{G}_{0} \mathrm{~W}_{0}$ bandgaps of (a) Pmma-SiS and (b) silicene sulfide as functions of the inverse lengths of vacuum. 
IV. Band Structures of Pmma-SiS, two layer Pma2-SiS and two layer silicene sulfide
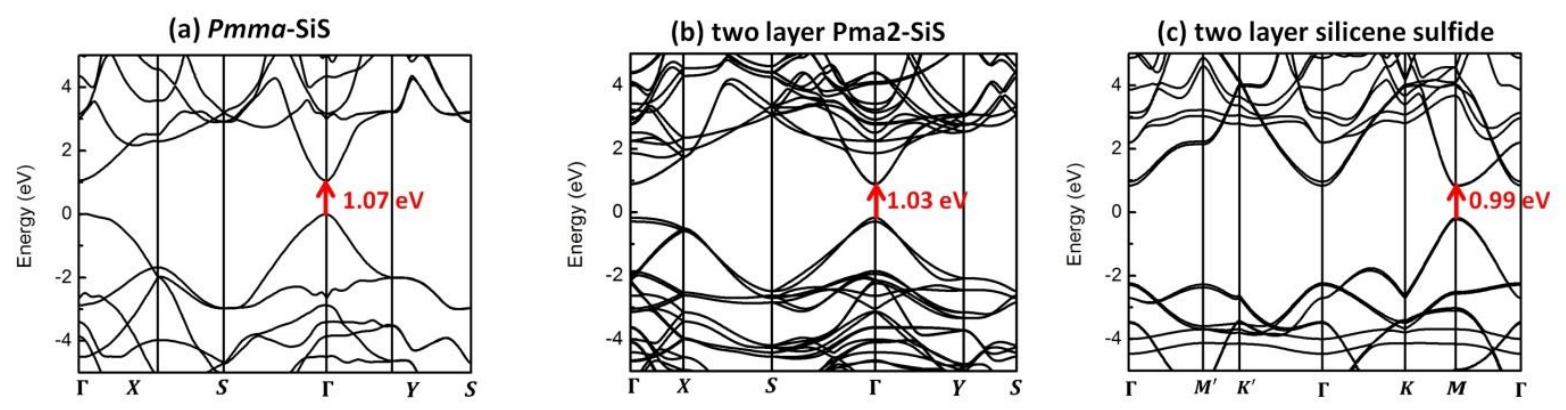

Fig. S4. Band structures of (a) Pmma-SiS, (b) AA-stacking two layer Pma2-SiS and (c) AAstacking two layer silicene sulfide. 


\section{Deformation Potential Constants and Elastic Modulus}
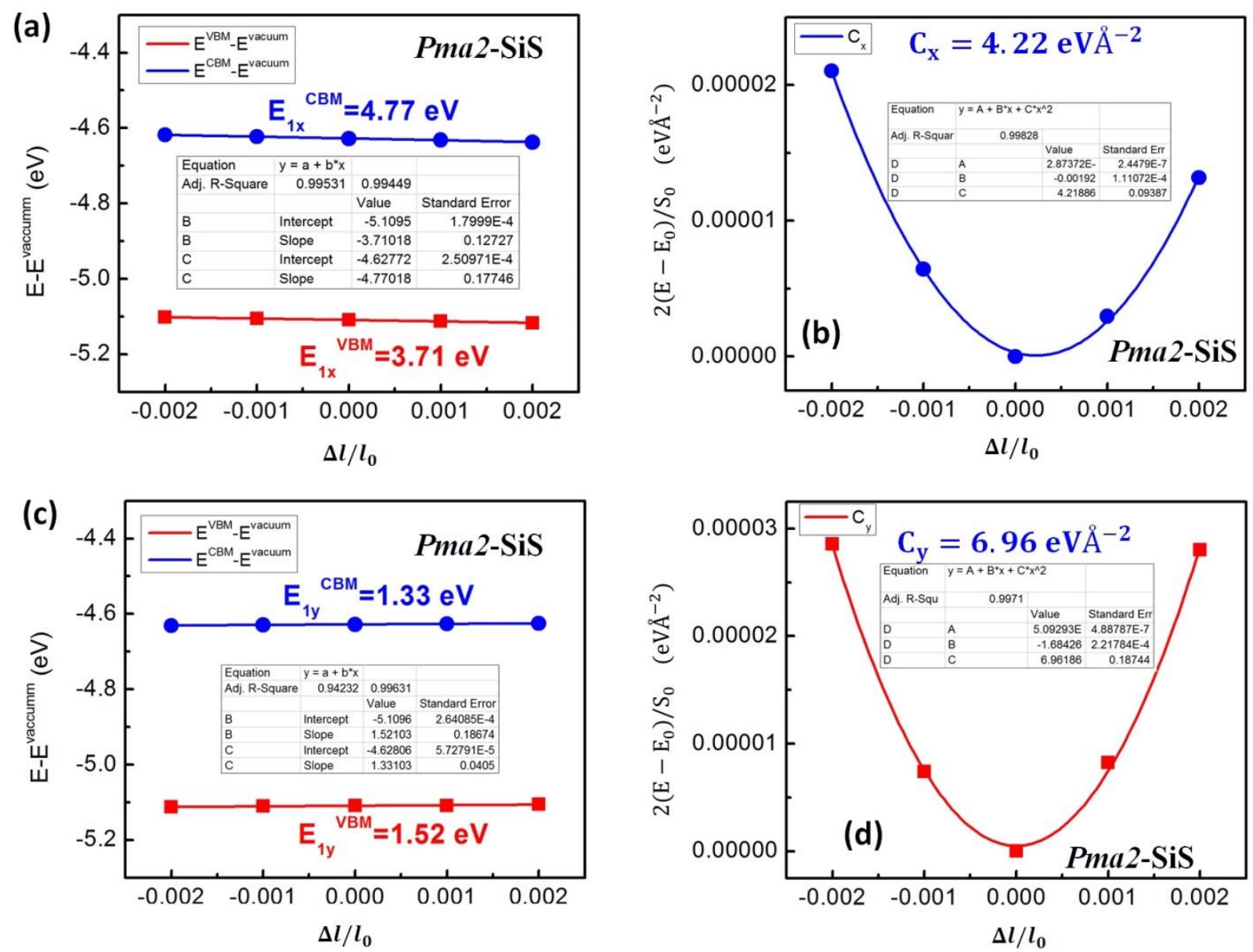

Fig. S5. (a) Deformation potential constants and (b) elastic modulus along Si zigzag chain direction in Pma2-SiS. (c) Deformation potential constants and (d) elastic modulus along Si-S-Si bond direction in Pma2-SiS. 

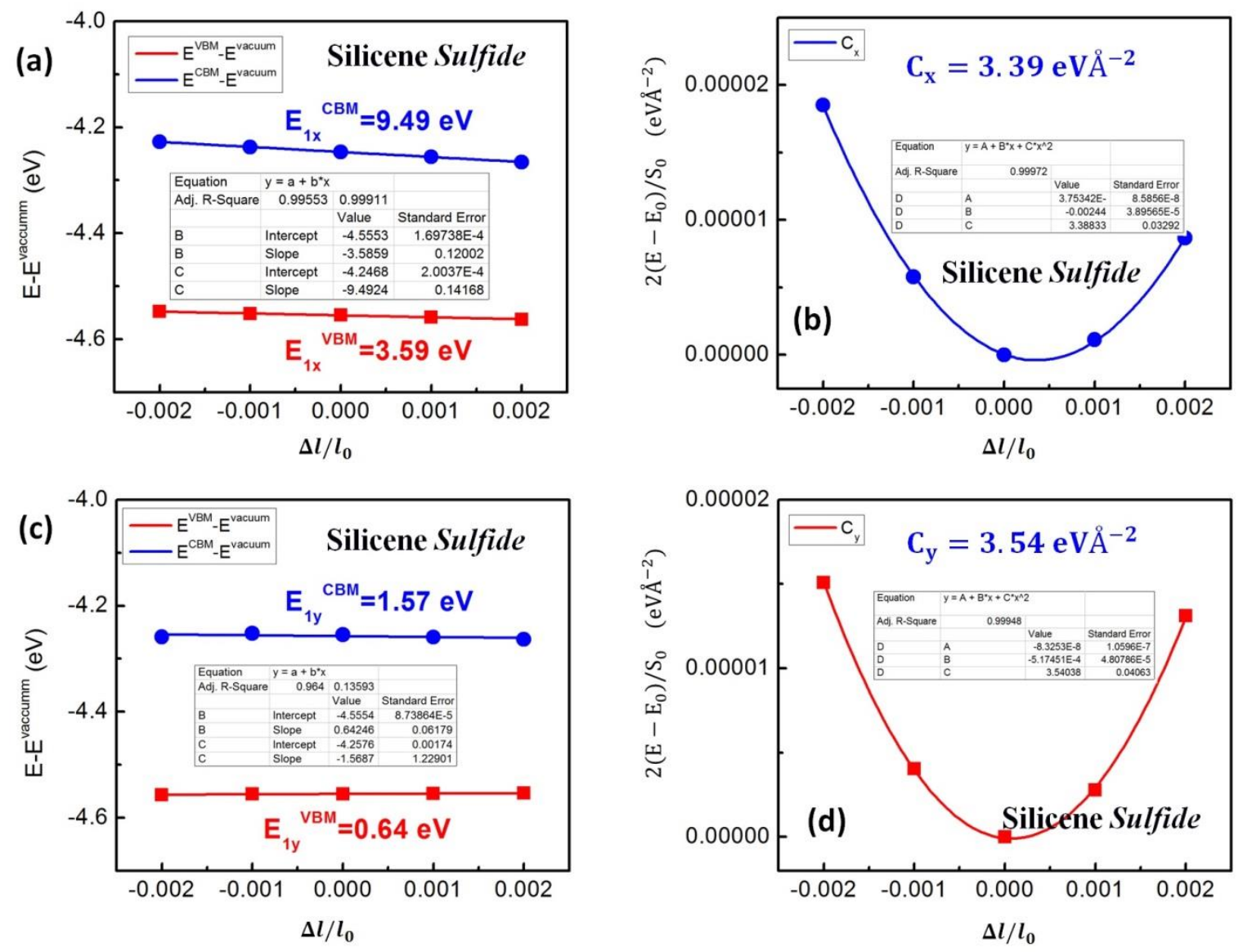

Fig. S6. (a) Deformation potential constants and (b) elastic modulus along Si zigzag chain direction in silicene sulfide. (c) Deformation potential constants and (d) elastic modulus along Si-S-Si bond direction in silicene sulfide. 


\section{Mobility Calculations from Conductivity}

The electrical current of carriers is defined as:

$$
\vec{J}=e \sum_{i, \vec{k}} f_{i, \vec{k}} \vec{v}_{i, \vec{k}}=\sigma \cdot \vec{E},(S 1)
$$

where $e$ is the charge of carriers, $(i, \vec{k})$ is band index and $\mathrm{k}$ point, $f_{i, \vec{k}}$ is the population of quantum state $(i, \vec{k})$, and $\vec{v}_{i, \vec{k}}$ is the group velocity defined as: $\vec{v}_{i, \vec{k}}=\frac{1}{\hbar} \frac{\partial \varepsilon_{i, \vec{k}}}{\partial \vec{k}}$, which can be obtained from band structures. To obtain $f_{i, \vec{k}}$, we need to solve the Boltzmann's equation:

$$
\frac{\partial f_{i, \vec{k}}}{\partial t}=-\vec{v}_{i, \vec{k}} \cdot \frac{\partial f_{i, \vec{k}}}{\partial r}-\frac{e}{\hbar}\left(\vec{E}+\frac{\vec{v}_{i, \vec{k}} \times \vec{H}}{c}\right) \cdot \frac{\partial f_{i, \vec{k}}}{\partial \vec{k}}+\left(\frac{d f_{i, \vec{k}}}{d t}\right)_{\text {scatt. }}
$$

The first term on the right side is diffusion term, the second is field effect, and the third is scattering effect. Using the relaxation time approximation for the scattering term, and in the absence of fields and temperature gradients, the solution of the above equation is:

$$
f_{i, \vec{k}}=f_{0}\left(\varepsilon_{i, \vec{k}}\right)+e\left(-\frac{\partial f_{0}}{\partial \varepsilon}\right) \tau_{i, \vec{k}} \vec{v}_{i, \vec{k}} \cdot \vec{E},(S 3)
$$

where $f_{0}$ is Fermi distribution function which is dependent on electron chemical potential or Fermi level and temperature defined as: $f_{0}=\frac{1}{e^{\frac{\varepsilon-\mu}{k_{B} T}}+1}$. Combining Eqs. (1) and (3) and considering the first term of right side of Eq. (3) has no contribution to the conductivity when the sum runs over whole reciprocal space, we can obtain the electrical conductivity as:

$$
\sigma=e^{2} \sum_{i, \vec{k}}\left(-\frac{\partial f_{0}}{\partial \varepsilon}\right) \tau_{i, \vec{k}} \vec{v}_{i, \vec{k}} \vec{v}_{i, \vec{k}},(S 4)
$$

When considering the transport anisotropy, the conductivity is a tensor:

$$
\sigma_{\alpha, \beta}=e^{2} \sum_{i, \vec{k}}\left(-\frac{\partial f_{0}}{\partial \varepsilon}\right) \tau_{i, \vec{k}} \vec{v}_{i, \vec{k}}^{\alpha} \vec{v}_{i, \vec{k}}^{\beta}(S 5)
$$


In principle, the relaxation time $\tau_{i \vec{k}}$ is dependent on the band index and $\mathrm{k}$ point, but in practice, it doesn't change much for different bands and k points. As a result, the relaxation time is usually approximated as a constant, which is known as constant relaxation time approximation. The conductivity then can be calculated from band structures with an assumed relaxation time. Detailed description of the calculations of electrical conductivity can be found in PRB, $\mathbf{6 8}$, 125210 (2003) and Comput. Phys. Commun. 2008, 178, 685-699. The carrier density can be easily obtained from integrating the DOS times the Fermi distribution function at given electron chemical potential and temperature. Here the effect of minority carriers is neglected (Comput. Phys. Commun. 2008, 178, 685-699) and the carrier density is given by:

$$
\begin{gathered}
n_{0}=\int_{E_{g}}^{\infty} d \varepsilon\left[1+e^{\frac{\varepsilon-\mu}{k_{B} T}}\right]^{-1} D(\varepsilon), \quad \text { if electron is the major carrier, } \\
p_{0}=\int_{-\infty}^{0} d \varepsilon\left\{1-\left[1+e^{\frac{\varepsilon-\mu}{k_{B} T}}\right]^{-1}\right\} D(\varepsilon), \text { if hole is the major carrier, }(S 6)
\end{gathered}
$$

where $D(\varepsilon)$ is the electron density of state and zero energy point is set at the VBM. Finally, we can obtain the mobility from $\mu=\sigma / e n$, which is dependent on the assumed relaxation time constant. In this work, we fitted this constant from the experimental measured mobility of phosphorene, which is about $250 \mathrm{fs}$. 


\section{Band Structures Changes with Strains}
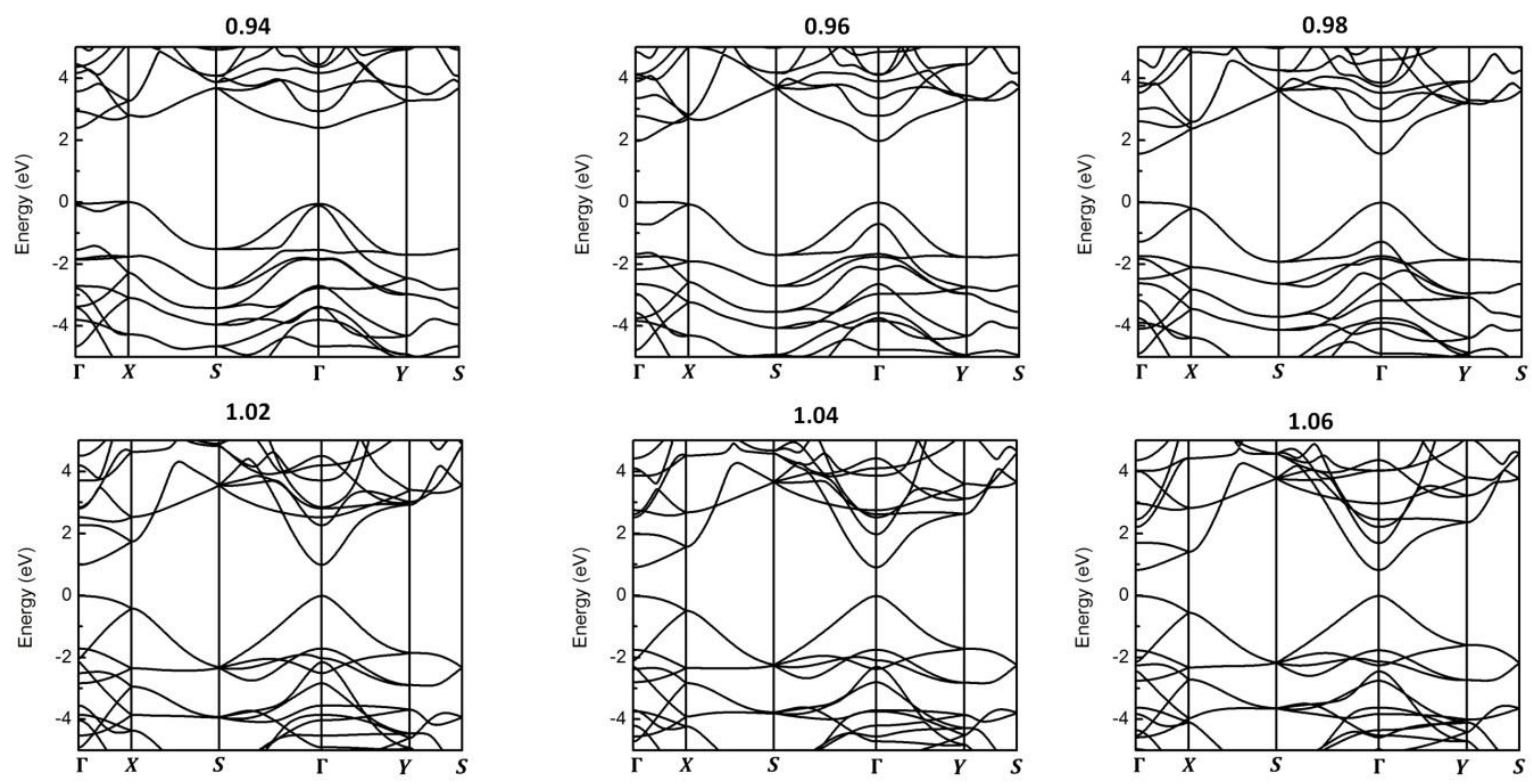

Fig. S7. (a) Band structure changes with biaxial strains in Pma2-SiS.
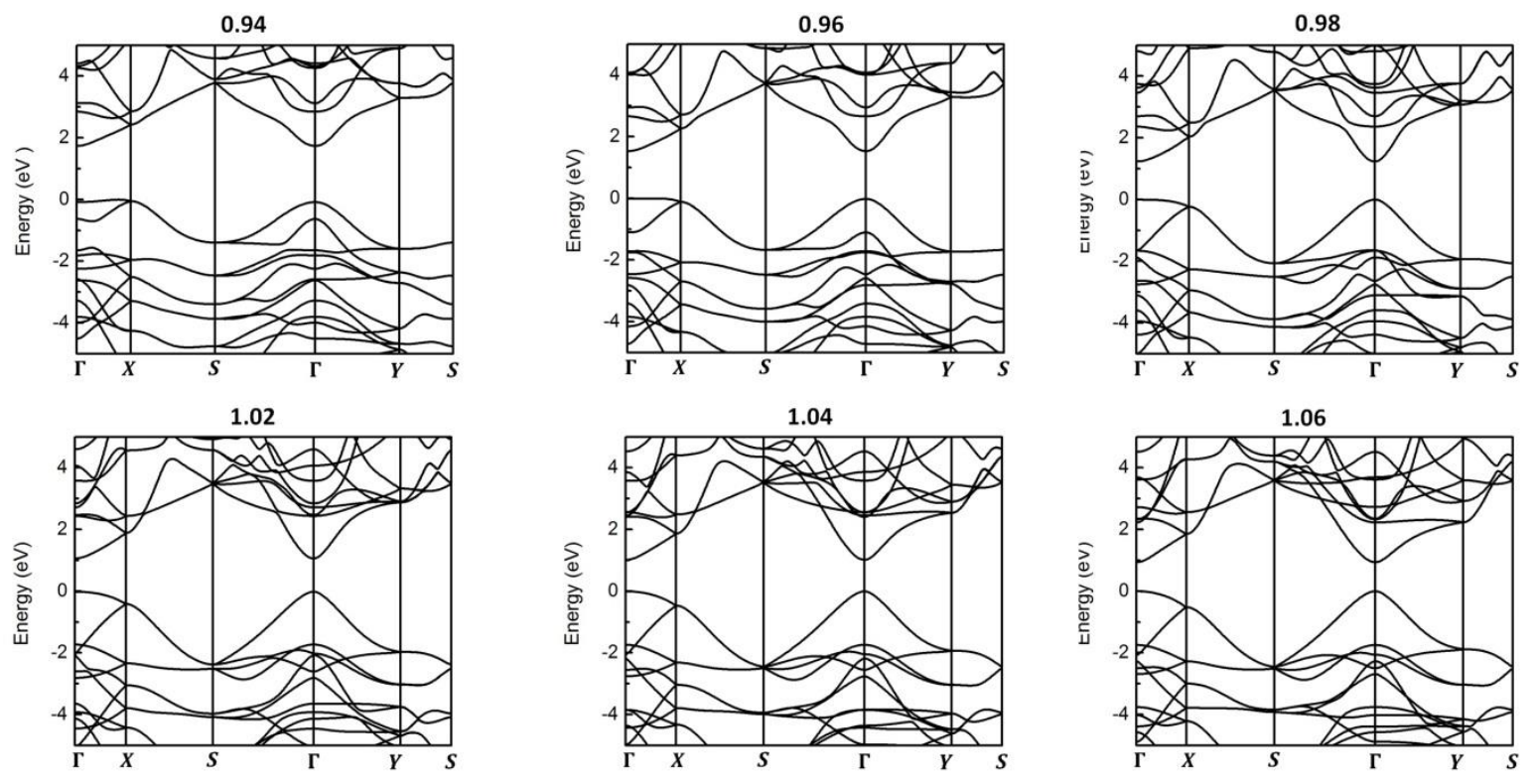

Fig. S8. (a) Band structure changes with $a$-direction strains in Pma2-SiS. 

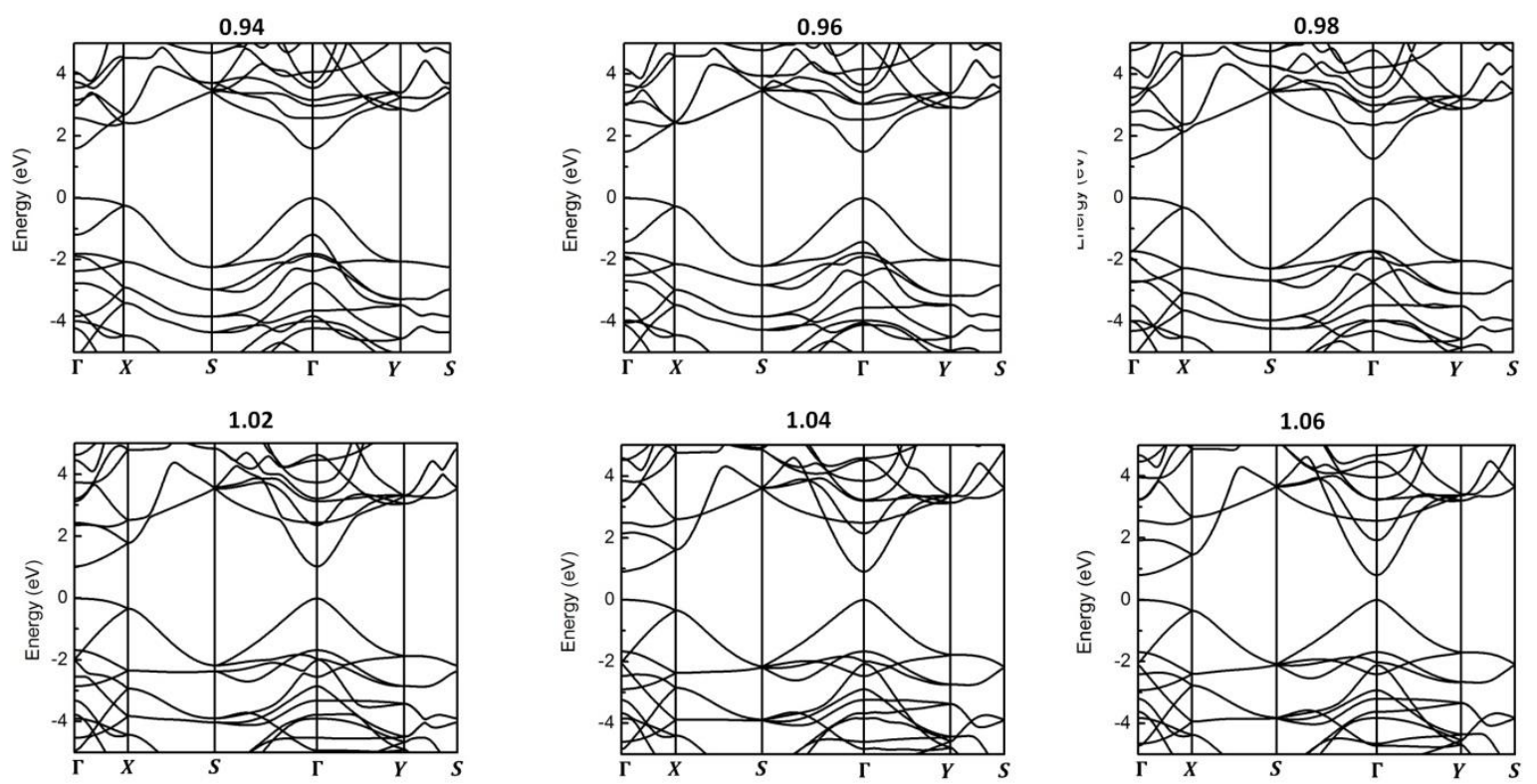

Fig. S9. (a) Band structure changes with $b$-direction strains in Pma2-SiS.
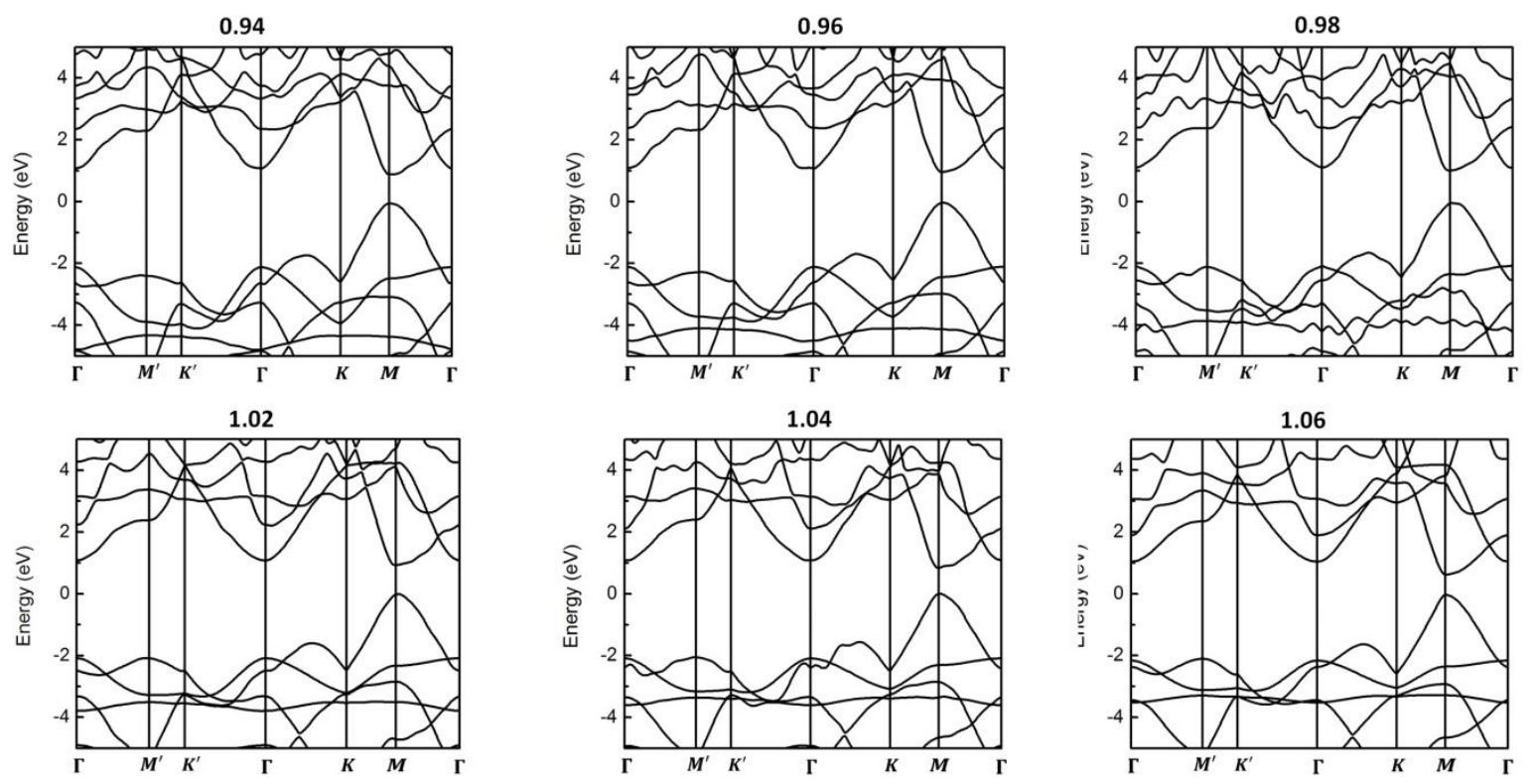

Fig. S10. (a) Band structure changes with biaxial strains in silicene sulfide. 

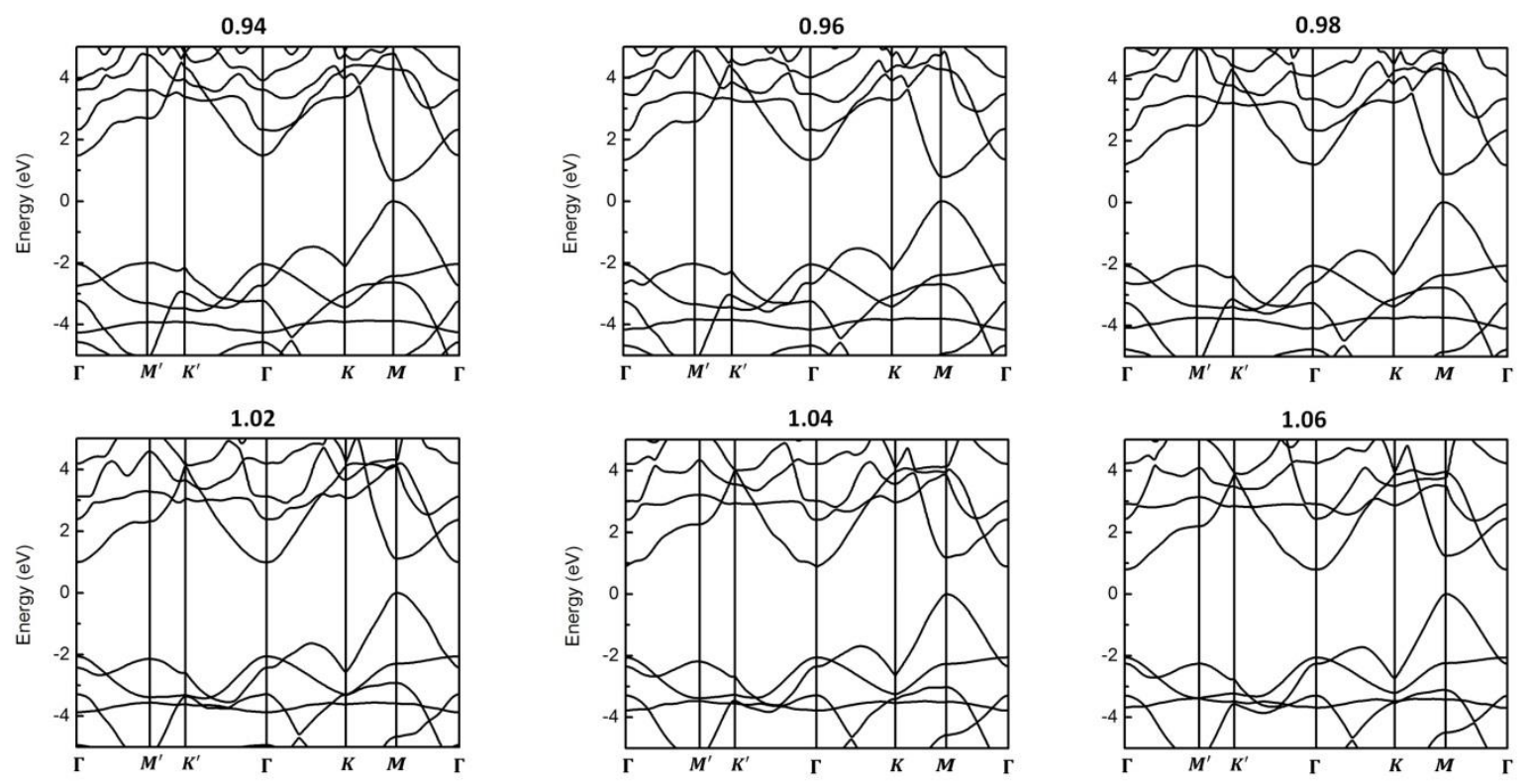

Fig. S11. Band structure changes with $a$-direction strains in silicene sulfide.
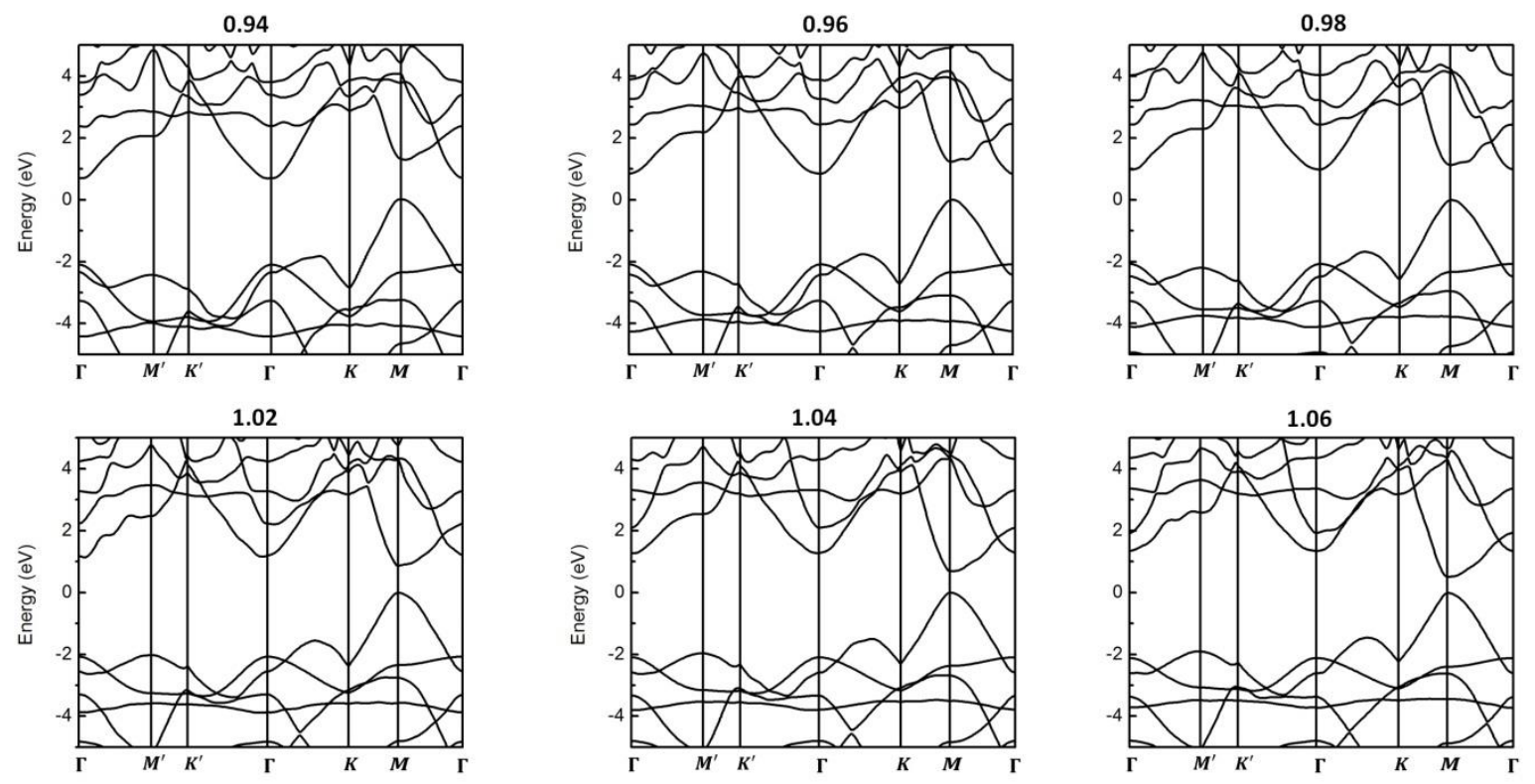

Fig. S12. Band structure changes with $b$-direction strains in silicene sulfide. 


\section{Atomic configurations and density of states of $O$ interstitials and $O$ substituting $\mathrm{S}$ in Pma2-SiS.}
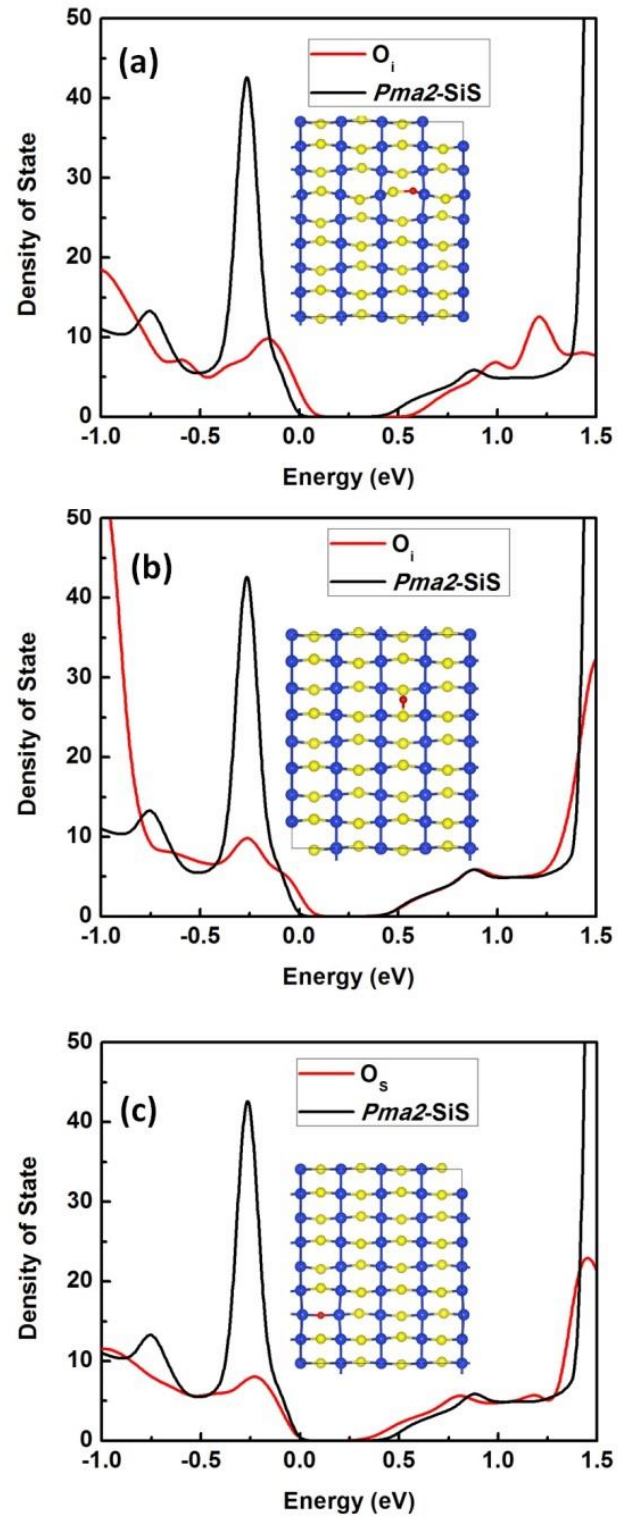

Fig. S13. Atomic configurations and density of states for (a) and (b) $\mathrm{O}$ interstitials, and (c) $\mathrm{O}$ substituting S in Pma2-SiS. 